\title{
Meeting frameworks must be even more inclusive
}

To the Editor - Inclusivity in science, technology, engineering and mathematics (STEM) is paramount and must include scientists with disabilities. One critical element to ensure inclusivity is accessibility at conferences, meetings and workshops. At these gatherings, scientists of all career stages present, listen and network in order to advance science. A recent article set out a new framework and guidance for inclusive and sustainable science, but it did not consider the needs of scientists with disabilities ${ }^{1}$. Diversity is not only defined as gender and cultural diversity, and many forget that it also includes disability. Any framework and guidance for conferences, meetings and workshops must consider and include accessibility and disability and their efforts to diversify science ${ }^{2}$. For instance, several STEM professional societies, including the Ecological Society of America, Association for the Sciences of Limnology and Oceanography ${ }^{3}$ and Mathematics Association of America, are currently taking the lead in including scientists with disabilities in conversations through organizing chat and panel events and publishing blogs and bulletins.

Moreover, sustainable conference planning must include intersectionality. For instance, two dimensions of diversity race and disability - cannot be separated. Ableism (oppression based on disability status) is inherently anti-Black, as ideas about disability and normalcy are rooted in racism ${ }^{4}$. Black people and other people of colour are more likely to be disabled or perceived as disabled, and in turn experience oppression, ableism and exclusion. Inclusion in STEM cannot be addressed without also addressing racial equity and inclusion. Simply aiming for conference attendees from all continents does not account for the intersectional oppression of Black and disabled people globally. As the editors of this journal themselves wrote, collaborations and choices in science must not ignore race and must fight structural racism ${ }^{5}$.

The COVID-19 pandemic has moved many scientific conferences online indefinitely. Yet, the rush to shift online in response to the pandemic does not inherently mean that the events are more inclusive. There is a risk of ignoring the innovative strategies and best practices used for remote participation developed and used by disabled and deaf professionals ${ }^{6,7}$. Meeting organizers must learn from this existing expertise on inclusive meetings. This includes best practices for access on Zoom and other video conferencing software ${ }^{7}$, such as having a limited number of videos on at the same time including the interpreter, planning for access while organizing the event (for example, choosing platforms compatible with captioning and screen readers) and budgeting for professional access services (for example, captioning and sign language interpretation). Conference organizers should also take steps to increase the accessibility of posters and presentations. This could include requesting and enforcing guidelines for presenters in line with universal design principles, such as using plain language, avoiding flashing animations and presenting content in multiple modalities (for example, pairing audio with text). This includes uploading pre-recorded presentations in advance, leaving sufficient time for captioning before the material is posted, to ensure improved access for attendees, including both deaf scientists and non-native English speakers. Access needs will vary; other attendees may need alt-text or visual description, large-print copies or other formats.

Accessibility at scientific conferences, meetings and workshops is currently a major obstacle in the growth of the number of scientists with disabilities ${ }^{8}$. Despite major disability rights movements and legislation worldwide in the past several decades, there are still many barriers to the full inclusion of people with disabilities in science. Notably, these include both attitudinal and physical barriers. Nondisabled scientists often have negative perceptions and stereotypes about disabled people ${ }^{9}$; this stigma, lack of knowledge, and discomfort are partly responsible for disparities in conference accessibility - as well as education and hiring more broadly. For example, many disabled students feel unwelcome in STEM, yet strategies for addressing these gaps and improving student mentoring are achievable ${ }^{10}$.

We agree that the inclusion of diverse and inclusive groups is better for science. Ensuring that access is part of inclusion at conferences also benefits everyone. Disabled communities have exceptional innovation and problem-solving skills to bring to environmental sustainability. The broader scientific community benefits from new techniques that are developed because many scientific tools are not built with accessibility in mind. In the quest to organize conferences that are both environmentally and fiscally sustainable in a post-COVID-19 world, we must take a moment and analyse whether the mode (in-person or virtual), platform and places (online platforms or physical spaces) are accessible and ensure that the many elements of diversity, including disability and accessibility, are explicit dimensions of diversity and inclusion.

\section{Gabriela Serrato Marks (DD $1 \times$, Caroline Solomon (D) $2 \bowtie$ and Kaitlin Stack Whitney ${ }^{3 凶}$ \\ ${ }^{1}$ Earth, Atmospheric, and Planetary Sciences Department, Massachusetts Institute of Technology, Cambridge, MA, USA. ${ }^{2}$ School of Science, Technology, Accessibility, Mathematics and Public Health, Gallaudet University, Washington DC, USA. ${ }^{3}$ Science, Technology \& Society Department, Rochester Institute of Technology, Rochester, NY, USA.

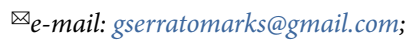 caroline.solomon@gallaudet.edu; kxwsbi@rit.edu}

Published online: 24 March 2021

https://doi.org/10.1038/s41559-021-01437-9

References

1. Blackman, R. C. et al. Nat. Ecol. Evol. 4, 668-671 (2020).

2. Goring, S. J., Stack Whitney, K. \& Jacob, A. L. Front. Ecol. Environ. 16, 63 (2018).

3. Solomon, C. Limnol. Oceanogr. Bull. 30, 20-21 (2021).

4. Lewis, T. A. Ableism 2020: an updated definition (25 January 2020); http://go.nature.com/3bDcFoO

5. Nat. Ecol. Evol. 4, 893-894 (2020)

6. Vogler, C. Accessibility tips for a better Zoom/virtual meeting experience. Gallaudet University Technology Access Program $(23$ March 2020); http://go.nature.com/3bDcR7w

7. Leary, A. How to make your virtual meetings and events accessible to the disability community. Rooted in Rights (13 April 2020); http://go.nature.com/3bCnGXg

8. Serrato Marks, G. How to make professional conferences more accessible for disabled people: guidance from actual disabled scientists. Union of Concerned Scientists Blog (8 November 2018); https://go.nature.com/3qFFV2i

9. Woodcock, K., Rohan, M. J. \& Campbell, L. High. Educ. 53, 359-379 (2007).

10. Braun, D. C. et al. CBE: Life Sci. Educ. 17, es10 (2018).

Competing interests

The authors declare no competing interests. 